\title{
Geographic Patterns in the Evolution of Resistance and Virulence in Drosophila and Its Parasitoids
}

\author{
A. R. Kraaijeveld ${ }^{\star}$ and H. C. J. Godfray
}

Natural Environment Research Council Centre for Population Biology and Department of Biology, Imperial College at Silwood Park, Ascot, Berkshire SL5 7PY, United Kingdom

AвSTRACT: Many insects are attacked by internal parasitoids against which they mount a largely cellular immunological defense. The resistance of a host and the virulence of a parasitoid determine which species survives after parasitism. Drosophila is parasitized by several hymenopterous parasitoids, especially those in the genera Asobara and Leptopilina. Geographic patterns have been found in parasitoid virulence and host resistance, the clearest of which is a cline in Asobara tabida virulence from the north (low) to the south (high) of Europe. Drosophila melanogaster resistance is highest in centralsouthern Europe and lower elsewhere. We review and interpret these patterns in the light of recent experimental and theoretical studies of the evolution and coevolution of these traits. We find no evidence for genotype-specific virulence and defense, which makes "Red Queen"-type coevolution unlikely. The most important explanation for the patterns is geographic differences in host-parasitoid community structure. Asobara tabida virulence is positively correlated with the resistance of its main hosts, and there is more limited evidence that $D$. melanogaster resistance is influenced by the virulence of its parasitoids. We critically appraise whether the evidence available so far supports a coevolutionary explanation for the levels of these traits.

Keywords: coevolution, resistance, virulence, Drosophila, parasitoids, geographic variation.

As Thompson $(1994,1999$, in this issue) has stressed, studies of coevolutionary interactions have tended to focus on single populations or species, neglecting the complex interactions that are likely to occur between the individual populations of species that occupy a wide geographic range, each of which experiences different biotic and abiotic selection pressures. In this article we discuss possible coevolutionary interactions between Drosophila and its * To whom correspondence should be addressed; E-mail: a.kraayeveld@
ic.ac.uk.

Am. Nat. 1999. Vol. 153, pp. S61-S74. (c) 1999 by The University of Chicago. 0003-0147/99/5305S-0005\$03.00. All rights reserved. parasitoids, concentrating on patterns of resistance and virulence over geographic scales. After introducing the system and briefly outlining the physiology of the host-parasitoid interaction, we describe the known geographic patterns of host resistance and parasitoid virulence, in particular looking for clines and mosaics. In the next section we consider three classes of mechanism that may explain these patterns: interactions between tightly coupled pairs of hosts and parasitoids, abiotic influences, and the effect of the wider host-parasitoid community. We finish by critically appraising the evidence for multispecies and more tightly coupled forms of coevolution in this system and by suggesting how the presence of coevolutionary interactions may be further explored. Throughout we concentrate on Drosophila and its parasitoids because with the exception of the very important work of Henter and Via (1995) and Henter (1995) on an aphid-parasitoid system, nearly all work in this area concerns Drosophila (see Kraaijeveld et al. 1998, for a review).

Parasitoids are insects that lay their eggs on or in the bodies of other insects and whose larvae eventually kill their host (Godfray 1994). Their lifestyle is thus intermediate between true parasites and predators. In this article we shall be concerned almost exclusively with parasitoids that feed internally and that remain quiescent while their host grows to full size (koinobiont endoparasitoids). During this quiescent stage, the parasitoid is exposed to the host's immune defenses, which it tries to inactivate. There is thus ample scope for classical antagonistic, "arms race" coevolution, though note an inherent asymmetry: whereas every parasitoid must overcome its host's defenses, not all hosts will suffer parasitoid attack.

The most important defense mechanism of insects against parasitoids and other foreign bodies that are too large to be phagocytosed is a cellular immune response called encapsulation. Certain hemocytes recognize a parasitoid egg or larva as foreign and rupture, causing other hemocytes to aggregate and form a capsule around the foreign body. The constitutive cells of the capsule fuse, and a cascade of biochemical reactions leads to the deposition of melanin and the hardening of the capsule (see, 
e.g., Gillespie et al. 1997). The parasitoid dies from suffocation or because of the release of necrotizing compounds. Parasitoids have evolved many adaptations to outsmart the host defenses, ranging from passive strategies, such as molecular mimicry or concealment from circulating hemocytes, to the active destruction of the host's immune system using toxins or symbiotic viruses (Strand and Pech 1995). The terms "resistance" and "virulence" are used in varying ways in different areas of parasitology; in the present context, "resistance" is the host's ability to survive attack by the parasitoid, and "virulence" is the parasitoid's ability to defeat the host's defenses.

We concentrate here on European Drosophila species that feed on fermenting substrates. These are members of the melanogaster group (Drosophila melanogaster and Drosophila simulans) and the obscura group (six sibling species of which Drosophila subobscura is the commonest; Kraaijeveld and van der Wel 1994). Parasitoids are known to be a major cause of larval mortality of these species in the field (Carton et al. 1986; Janssen et al. 1988; Driessen et al. 1990), and they belong to two main guilds: internal larval parasitoids and pupal parasitoids.

The most important larval parasitoids are the braconid Asobara tabida and the two eucoilids Leptopilina heterotoma and Lepropolina boulardi. Asobara tabida attacks mainly hosts in fermenting substrates and is common across most of Europe (though it appears to be lacking from most of the Iberian peninsula; Kraaijeveld 1994). Leptopilina heterotoma is rather a generalist, attacking Drosophila larvae in a variety of substrates (Janssen et al. 1988) and is found throughout Europe (although it appears to be less common in the eastern Mediterranean; Kraaijeveld 1994); L. boulardi is a specialist on larvae of the melanogaster group and, in Europe, is only found in the Mediterranean. The two genera of larval parasitoids have different strategies for overcoming host defenses (Carton and Nappi 1997). Although the details are not fully resolved, an important mechanism in Asobara is concealment of eggs by adhesion to host tissue such as fat body. This adhesiveness results from a fibrous development of the egg exochorion (Eslin et al. 1996). Leptopilina, on the other hand, injects at the time of parasitism viral-like particles composed of protein but not DNA. Initially embedded in the folds of the egg chorion, they are released into the hemocoel as the egg swells inside the host and the chorion unfolds. In a manner that is not understood, the particles appear to induce hemocyte apoptosis and hence prevent encapsulation (Rizki and Rizki 1990; Dupas et al. 1996).

Drosophila pupae are attacked by several species in the pteromalid genera Pachycrepoideus (by far the commonest) and Spalangia and by the diapriid genus Trichopria (Carton et al. 1986; Kraaijeveld 1994). As will be discussed in more detail later, most of these species feed between the pupal and puparial walls and are thus ectoparasitoids and do not come into contact with the host's immune system. Potential defense mechanisms against this type of parasitoid include thickening of the puparial wall and/or shortening of the pupal period to minimize the window of vulnerability.

\section{The Patterns}

\section{Geographic Variation in Parasitoid Virulence}

Geographic variation in Drosophila parasitoid virulence was first discovered by Walker $(1959,1962)$, who found a large difference between two Swiss populations of Leptopilina heterotoma in their ability to prevent encapsulation by Drosophila melanogaster. More recently, studies of Leptopilina boulardi populations from different regions of Europe and even different continents have revealed marked differences in virulence against both sympatric and allopatric strains of fly (Carton 1984; Boulétreau 1986; Carton and Nappi 1991). We shall return later to Carton's (1984) suggestion that these data show evidence of local adaptation.

Asobara tabida virulence has been surveyed across the whole of Europe (Kraaijeveld and van Alphen 1994). Virulence was measured as the ability of $A$. tabida to survive encapsulation in a standard strain of fly that originated from Hamburg, Germany. As we shall describe later, host resistance to $A$. tabida appears to be a one-dimensional trait, and the choice of standard strain with which to assess the wasp influences only the mean virulence and not the rank order of different wasp populations. In Europe, there is a strong and clear north-south cline in parasitoid virulence (fig. 1): parasitoids from northern, western, and central Europe have a low virulence (typically about 20\%), whereas parasitoids from the Mediterranean have a much higher virulence (60\%-95\%; Kraaijeveld and van Alphen 1994).

Exceptions to this trend may provide clues to the mechanisms responsible. Two northern European strains of $A$. tabida, collected in habitats associated with humans (a tropical bird shop and an indoor fruit market; Kraaijeveld 1994) had a virulence intermediate between typical northern and Mediterranean field strains (35\%-61\%). In addition, a comparison of strains collected in (semi-) natural and urban habitats in the Netherlands showed that strains from urban habitats had a slightly (but significantly) higher virulence than their conspecifics from more natural habitats (29\% vs. 19\%; Kraaijeveld 1994). More surprisingly, very high virulence $(94 \%)$ was found in a strain collected in Scotland from the slime on sherry casks (Kraaijeveld 1994). Two A. tabida strains collected in western Canada showed a virulence of about $40 \%$, intermediate between 


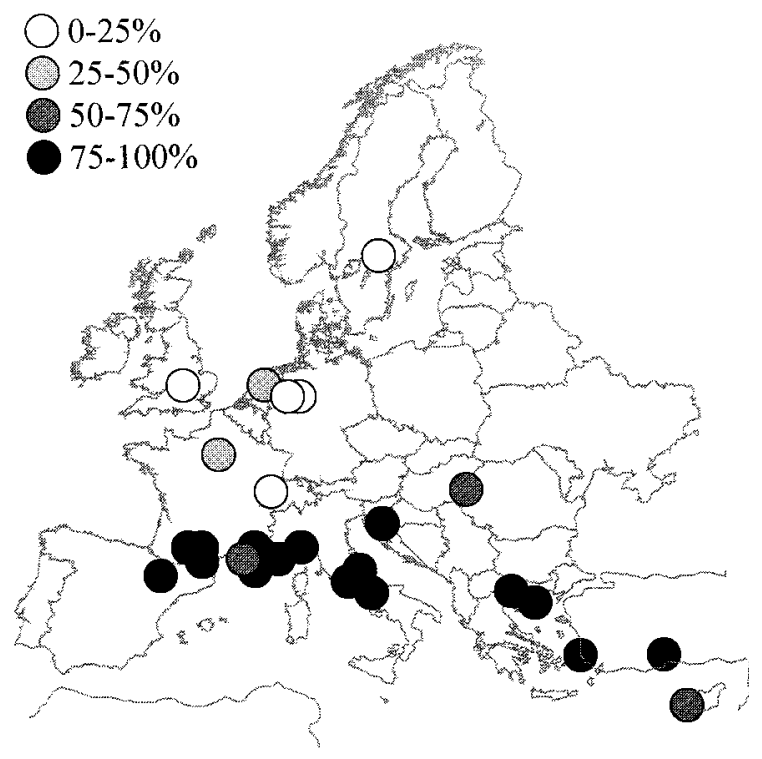

Figure 1: Geographic patterns across Europe in the virulence of field populations of Asobara tabida against Drosophila melanogaster. The symbols represent different levels of the percentage of eggs escaping encapsulation in a test strain of host.

typical northern and southern European strains (Kraaijeveld and van Alphen 1994). These results suggest virulence may be influenced by complex local factors superimposed on broad geographic trends.

The cline in virulence is correlated with the passive defense mechanism of this parasitoid. It has been found that eggs from Mediterranean strains of A. tabida have a "sticky" chorion and so become embedded more readily in host tissue than the eggs of northern strains (Kraaijeveld and van Alphen 1994).

\section{Geographic Variation in Host Resistance}

The earliest studies of encapsulation ability in D. melanogaster found marked differences in populations collected at different sites (Oprecht and Hadorn 1952; SchlegelOprecht 1953; Walker 1959; Hadorn and Walker 1960; Hadorn and Graßmann 1962). This was confirmed by the French school (Carton, Boulétreau, and coworkers), who found, for example, that D. melanogaster from the Congo performed better against different $L$. boulardi strains than flies from southern France, which in turn were superior to Tunisian flies (Boulétreau and Fouillet 1982; Carton and Boulétreau 1985; Wajnberg et al. 1985; Boulétreau 1986). Carton and Nappi (1991) compared the performance of five $D$. melanogaster populations against sympatric L. boulardi strains and found low encapsulation rates with the exception of flies from the Congo.
Drosophila melanogaster resistance against $A$. tabida has been surveyed on a Europe-wide basis (fig. 2; Kraaijeveld and van Alphen 1995). Resistance was measured against a standard strain, originating from southern France. The geographic pattern is more complex than that for A. tabida virulence. Resistance is strongest in central-southern $\mathrm{Eu}$ rope but weaker in the north, in the Iberian peninsula, and in the southeast.

On a European scale, D. melanogaster populations also show geographic variation in resistance against $L$. boulardi. However, the pattern is rather mosaic (fig. 3; Kraaijeveld and van Alphen 1995), and there is no correlation across geographic strains between resistance against $L$. boulardi and A. tabida (fig. 4).

\section{Explanations}

\section{Single-Species Interactions}

Parasitoids are an important mortality source for many of their hosts, which means both that selection on the traits of resistance and virulence will normally be strong and that parasitoids are likely to have an important impact on host population dynamics. In this section we explore whether a coupled interaction between a single host and parasitoid species can generate the type of patterns described in the last section. We first explore the nature of the two traits, then look at the evidence for additive genetic

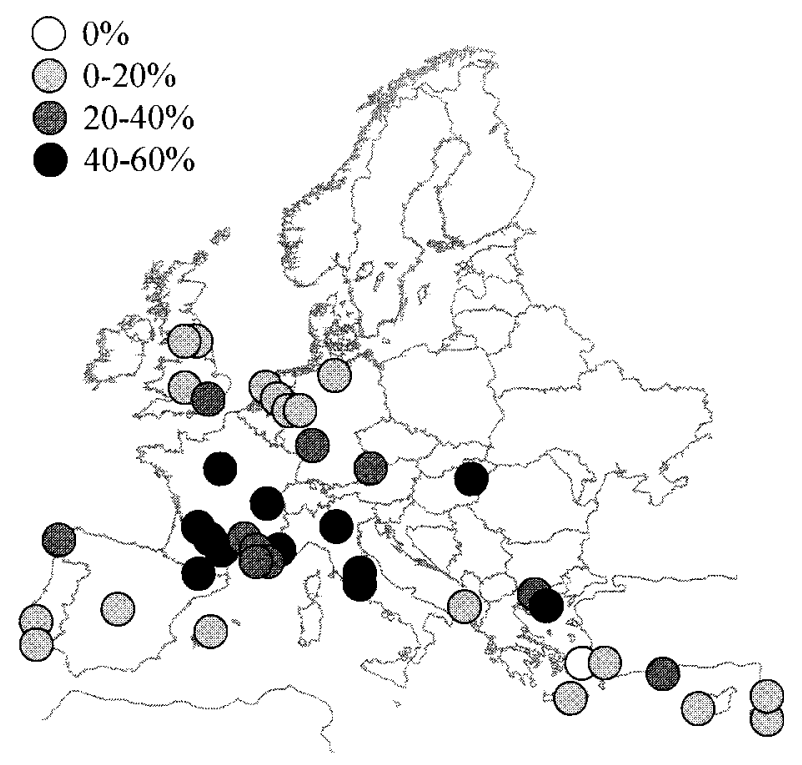

Figure 2: Geographic patterns across Europe in the encapsulation ability of field populations of Drosophila melanogaster against Asobara tabida. The symbols represent different levels of the percentage of eggs encapsulated when attacked by a test strain of parasitoid. 


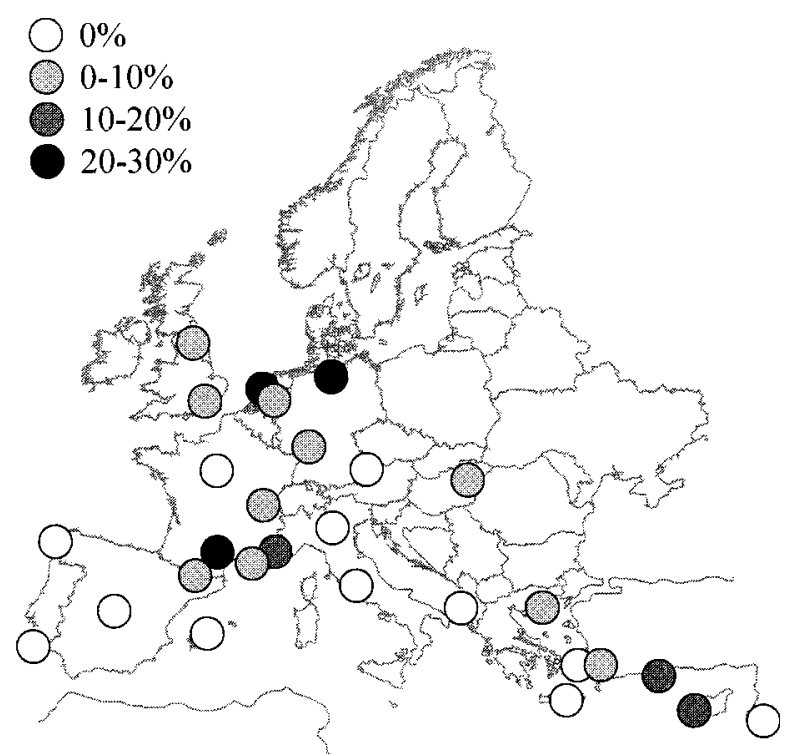

Figure 3: Geographic patterns across Europe in the encapsulation ability of field populations of Drosophila melanogaster against Leptopilina boulardi. The symbols represent different levels of the percentage of eggs encapsulated when attacked by a test strain of parasitoid.

variation in resistance and virulence and for resistance and virulence having costs. We also discuss the role of population dynamics and the few theoretical studies that have tried to integrate evolutionary and population dynamics to predict patterns of resistance and virulence.

Specificity and Local Adaptation. To understand the kind of selection pressure acting on resistance and virulence, it is important to understand the specificity of the immune response. Does selection for increased defensive ability raise resistance levels against all genotypes of a parasitoid species, or is resistance more specific? Similarly, are more virulent parasitoids successful against all host strains? This question is important, as specificity will give rise to adaptation to the most common genotype and hence a frequency-dependent advantage for rare genotypes. The result, as has been widely recognized (e.g., Thompson 1982), is locally dynamic or "Red Queen" coevolution with a constant flux of genotype frequencies and high heritability of virulence and resistance.

Carton (1984; see also Carton and Nappi 1991) studied five Drosophila melanogaster-Leptopilina boulardi associations from Europe, Africa, and South America and argued that their data were consistent with increased parasitoid success in sympatric associations. However, analysis of these data is complicated by high and variable postparasitism mortality in which both partners die. We reanalyzed the data (Kraaijeveld et al. 1998) and concluded that this jointly acting mortality was lower in sympatric associations, but given that the larva survives, the probability of the parasitoid winning did not differ between allopatric and sympatric associations. Further experimentation is needed to confirm these results, but they suggest local adaptation has occurred in a situation in which it benefits both the host and parasitoid.

We selected four D. melanogaster lines for increased resistance to a southern French strain of Asobara tabida (Kraaijeveld and Godfray 1997), raising their encapsulation ability from about 5\% to $60 \%$. When these lines were tested against three other strains of the same parasitoid species (from Leiden, the Netherlands; Kos, Greece; and Saanich, Canada), a clear difference remains between control and selection lines (fig. 5). Both the control and selected lines perform proportionately well on the very weak Canadian strain and proportionately worse on the very strong Greek strain. These data, and more informal experience with other parasitism experiments, suggest that the outcome of a novel host-parasitoid strain combination can be predicted by a knowledge of the general levels of resistance and virulence in the two partners, something that would not be possible if there were extensive local interactions.

Evidence for Genetic Variation. Whenever additive genetic variation in resistance in Drosophila has been sought, it has been found. The major evidence comes from isofemale line and selection experiments. Comparison of heterogeneity among isofemale lines provides a measure of a trait's heritability that is intermediate between narrow-sense and broad-sense heritability (Parsons 1980). French workers have used this technique on many different Drosophila populations (Boulétreau and Fouillet 1982; Carton and Boulétreau 1985; Wajnberg et al. 1985; Carton et al. 1989; Carton and Nappi 1991, 1992) with heritabilities as high as 0.43 recorded. Higher resistance in Drosophila was first obtained in artificial selection experiments by SchlegelOprecht (1953), Hadorn and Walker (1960), and Walker (1962) and more recently by others (Boulétreau 1986; Kraaijeveld 1994; Hughes and Sokolowski 1996; Kraaijeveld and Godfray 1997; Fellowes et al. 1998b). Fellowes et al.'s (1998b) study provides probably the most extreme example: over about six generations of selection, survival from $L$. boulardi attack in four lines of D. melanogaster increased from about $0.5 \%$ to $50 \%$.

In contrast, the evidence for additive genetic variation in parasitoid virulence is much weaker. Isofemale line studies have identified variation in L. boulardi virulence against Drosophila simulans (Carton et al. 1989; Carton and Nappi 1991), and there is a suggestion of higher virulence in populations of $L$. boulardi cultured together with D. melanogaster for many generations (Boulétreau 1986). 


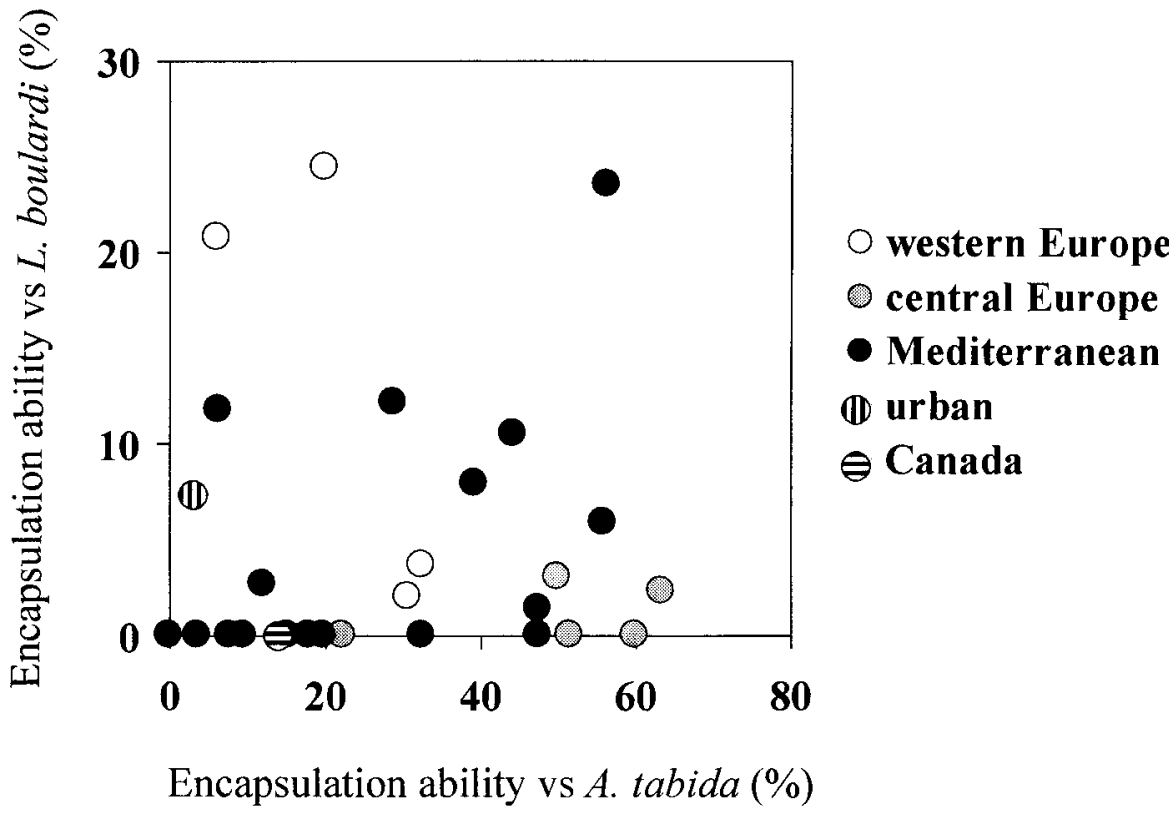

Figure 4: Correlation between the encapsulation ability of different populations of Drosophila melanogaster against test strains of Asobara tabida and Leptopilina boulardi. The symbols denote the geographic origin of the populations.

In ongoing experiments we have attempted to select artificially for increased virulence in five A. tabida lines, but at the time of writing (after nine generations), we have failed to achieve a significant increase.

In an important non-Drosophila study, Henter and Via (1995) found highly significant across-clone variation in parasitoid resistance in the pea aphid, Acyrthosiphon pisum, while Henter (1995) in a half-sib analysis found that its parasitoid Aphidius ervi (Braconidae) also possessed substantial additive genetic variation in virulence.

Costs and Benefits of Resistance and Virulence. In considering the costs of resistance, it is important to distinguish between two types of costs: the costs of mounting a defensive response and the costs of maintaining a defensive ability-analogous to the costs of waging war and maintaining a standing army. Given that parasitism has occurred, the host will be selected to invest whatever it takes to defeat the parasitoid, but if this action leads to a major reduction in fecundity or survival, it will slow the spread of the gene and make the maintenance of a defensive ability less worthwhile. In the case of parasitoid virulence, because all successful parasitoids must overcome host defenses, there is not the distinction between the two types of cost.

The costs of mounting a defense have been investigated by Carton and David (1983; against L. boulardi) and Fellowes et al. (1999; against A. tabida) in D. melanogaster.
Both studies found that individuals that had encapsulated a parasitoid were comparatively small and with female fecundity comparatively reduced. In addition, Fellowes et al. (1999) found a reduction in puparium thickness and a lower insemination success in males. Curiously, Pachycrepoideus vindemmiae, one of the pupal parasitoids of Drosophila, prefers pupae containing an encapsulated $A$. tabida egg compared with unparasitized pupae (Fellowes et al. 1998a), possibly because of the thinner puparial wall.

Demonstrating the maintenance costs of resistance is harder than doing so for the costs of mounting a defense. One technique is to use artificial selection to increase resistance and then to look for correlated reductions in other fitness components. It is important in such experiments to maintain high effective population sizes to reduce the risk of hitchhiking by deleterious recessive genes. We (Kraaijeveld and Godfray 1997) and Fellowes et al. (1998b) selected D. melanogaster for increased resistance against $A$. tabida and L. boulardi, respectively. In both cases, no differences were found in fitness components when control and selection lines were compared under conditions of plentiful resources. However, when competition for food became severe (as it often does in the field; Atkinson 1979), larvae from high-resistance lines were found to have reduced competitive ability compared with control lines (fig. 6). The fact that the same trade-off was found after selection for resistance against two different parasitoid species suggests that it may be a general cost of improved 


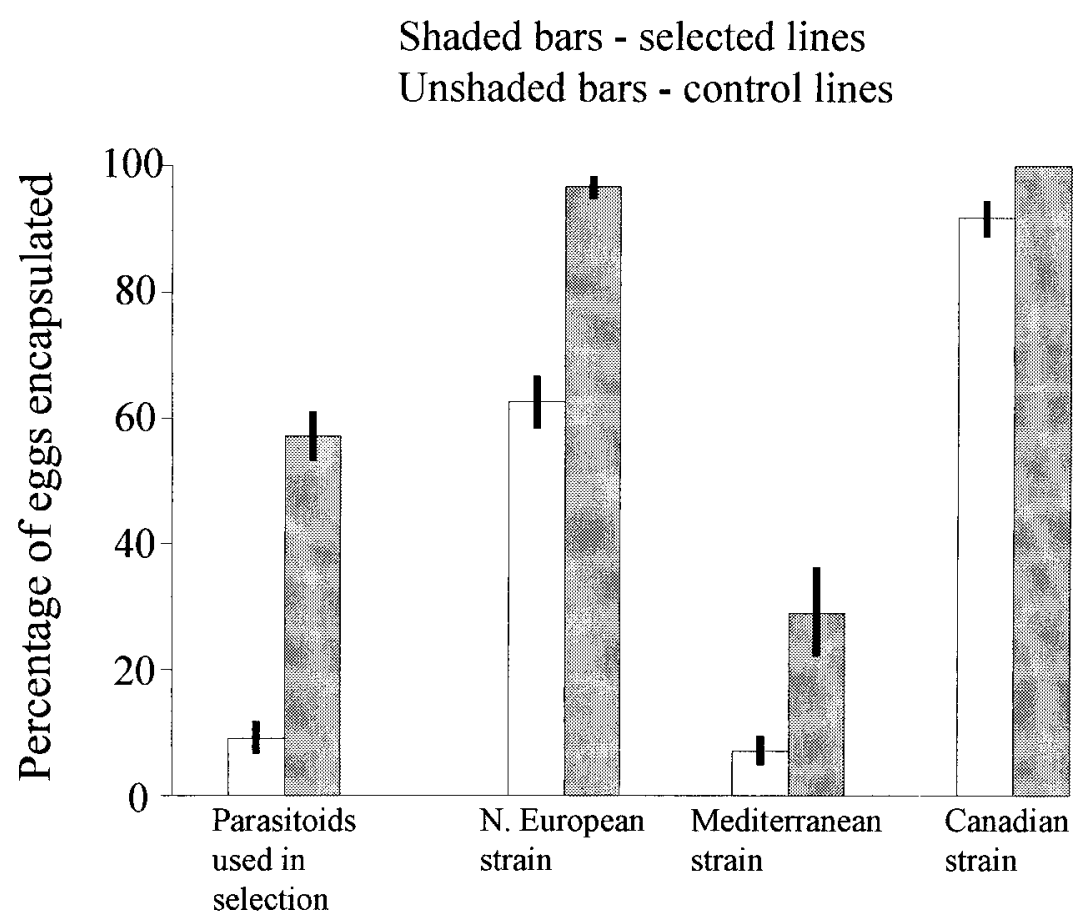

Figure 5: Encapsulation ability of Drosophila melanogaster lines selected for improved resistance against Asobara tabida when challenged by different strains of the same parasitoid species. The histograms represent means and SEs of four lines each of selected and control flies. The performance is shown against the wasp strain used in the selection experiments and three strains from different geographic regions. Note that selected flies always encapsulated Canadian wasps; hence, the $\mathrm{SE}=0$. All comparisons are significant at $P<.05$.

defense. When the selection pressure was removed and the lines were maintained under levels of competition of intermediate severity, the levels of resistance declined (Kraaijeveld et al. 1998), which again suggests the presence of a trade-off.

Population Dynamics and Theoretical Studies. Parasitoids always have to find hosts, but the latter may escape parasitism. The population dynamic factors that determine the probability of parasitism may thus be an important determinant of optimal levels of resistance and might potentially help explain the geographic patterns described earlier. Parasitism rates are known to vary widely in Drosophila species, and almost 100\% parasitism has been found on occasion (Carton et al. 1986; Janssen et al. 1988; Driessen et al. 1990). However, it is ironic that although host-parasitoid interactions are a model system in population ecology for investigating consumer resource interactions (see, e.g., Hassell 1978, 1998; Mills and Getz 1996; Murdoch and Briggs 1996; Godfray and Müller 1998), and the importance of Drosophila as a model system in evolutionary biology is undoubted (Powell 1997), there has been relative little study of Drosophila population dynamics in the wild (at least those species from fermenting substrates). Drosophila has probably proved unattractive to population dynamicists because of its use of ephemeral resources over a wide geographic area. A better understanding of Drosophila-parasitoid population dynamics is essential to untangling their coevolution.

Several early theoretical studies considered the influence of variable rates of encapsulation on population dynamic stability (Bailey et al. 1962; Hassell and Anderson 1984; Godfray and Hassell 1991), but only recently have workers attempted to model the joint population and evolutionary dynamics. Hochberg and Holt (1995) assumed that a fraction of the host population escaped parasitism and that this fraction was a function of the mean of a parasitoid trait (larger values decreasing the numbers escaping) and the mean of a host trait (larger values increasing the numbers escaping). Larger values of the parasitoid trait were assumed to reduce maximum attack rates, and larger values of the host trait were assumed to reduce fecundity. The population dynamics assumed were a modified form of the standard Nicholson-Bailey model of host-parasitoid population dynamics (Hassell 1978), and genetic change was incorporated using Lande's (1976) quantitative genetics model with constant genetic variation. The fraction escaping parasitism can be interpreted as the fraction of 


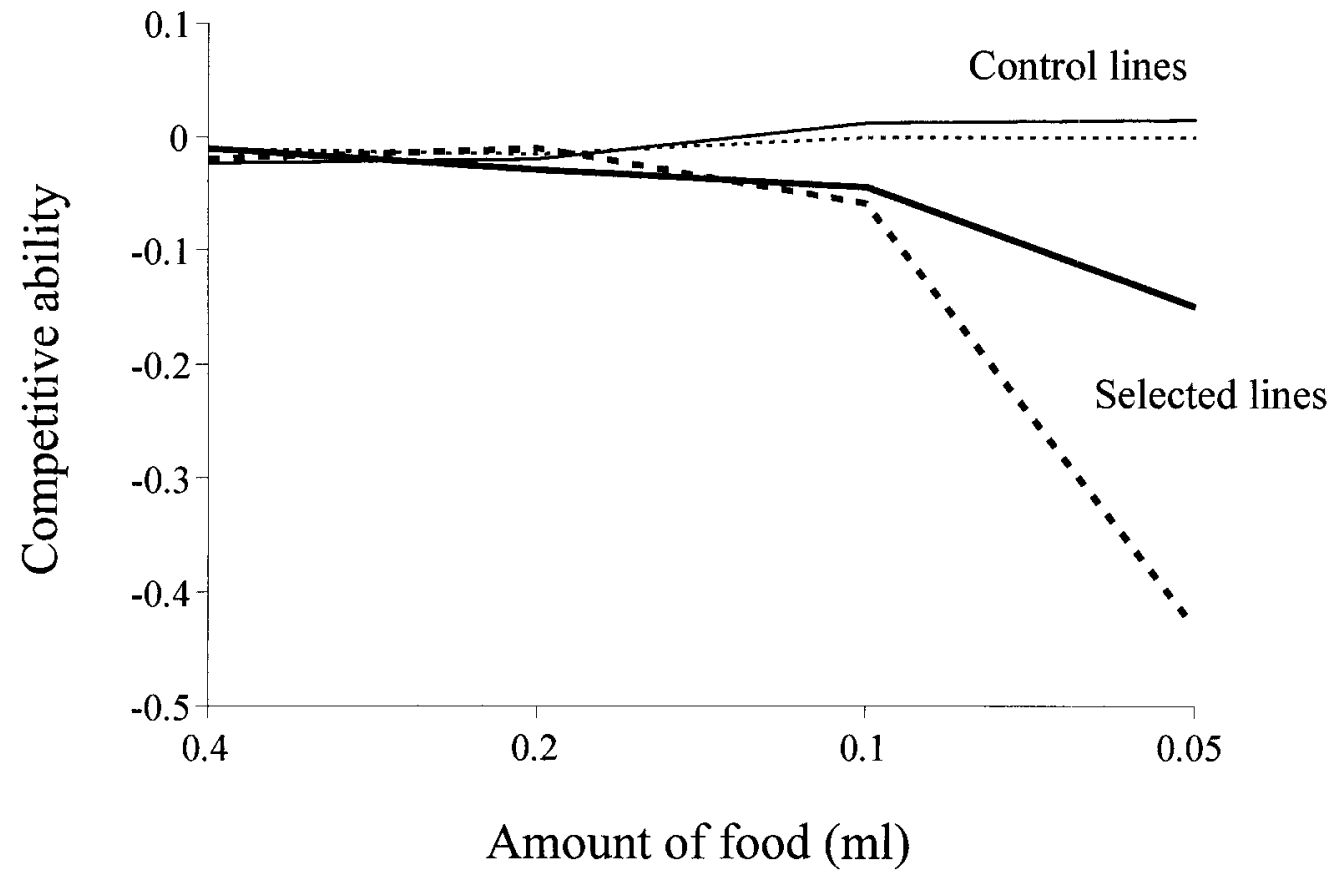

Figure 6: Competitive ability of Drosophila melanogaster larvae selected for increased encapsulation ability (thick lines; controls, thin lines) against Asobara tabida (solid lines) and Leptopilina boulardi (dashed lines) at different levels of food abundance (0.4, 0.2, 0.1 , and $0.05 \mathrm{~mL}$ food per 30 larvae). The SEs have been omitted for clarity (see Kraaijeveld and Godfray 1997 and Fellowes et al. $1998 b$ for full details of testing procedures). Selected lines have significantly lower competitive ability at $0.1 \mathrm{~mL}$ (both sets) and $0.05 \mathrm{~mL}$ ( L. boulardi lines; the A. tabida lines approach significance, $P=.06)$.

hosts able to encapsulate parasitoid eggs, though this formulation does not allow for specific interactions between different host and parasitoid genotypes and hence cannot show Red Queen coevolution. The results are complex, but their most important conclusions were that evolution could lead to changes in the population dynamic behavior of the system and that in addition to the standard population dynamic criteria, additional evolutionary conditions had to be met to allow a persistent interaction.

Doebeli (1997) also bases his population-dynamic model on the Nicholson-Bailey equations, and although he does not explicitly consider resistance and virulence, his model can be interpreted in this light. Suppose virulence and resistance are one-dimensional traits and that for a parasitoid to succeed its genotype must match that of its host. We are thus dealing with genotype-specific rather than quantitative mechanisms of resistance. Doebeli modeled resistance and virulence as multilocus traits and explicitly included sexual reproduction, which promotes genetic diversity. The model predicted genetic cycles in which over evolutionary time the parasitoid chases the host through the one-dimensional trait space, followed by the host jumping to a region where few parasitoids with matching genotypes exist. It is important that the refuges from parasitism generated by these genetic cycles were sufficient to stabilize the Nicholson-Bailey model, which otherwise displays divergent population oscillations, though sexual reproduction was essential for a persistent interaction and the trait space had to be large enough for some hosts to be protected from parasitoids. The basic model assumed the traits were not costly, though the broad conclusions were robust to the additions of costs.

Sasaki and Godfray (1999) took an approach similar to that of Doebeli (1997) except that they assumed resistance and virulence were linear traits with the probability of the host or parasitoid triumphing being proportional to the difference in investment in resistance and virulence. Like Doebeli, they found potential long-term genetic cycles, although for a subtly different mechanism: resistance and virulence increase in an arms race until a point is reached when resistance is so expensive that the host is selected to cease to defend itself, in effect gambling on not being discovered by the parasitoid. Given host surrender, the parasitoid is selected to reduce investment in virulence and the whole cycle starts again. For some parameter values, there is a stable genetic equilibrium, with hosts investing little or nothing in resistance and parasitoids investing moderately in virulence (some Drosophila species 
appear not to invest in resistance; see later discussion). The genetic cycles contributed toward population stability, but not as markedly as in Doebeli's model, though this may just reflect Sasaki and Godfray's assumption of asexual genetics.

These models suggest the interesting interactions involving resistance and virulence that may occur in both the population-dynamic and population-genetic spheres. However, further modeling requires a better understanding of the nature of these traits and of the dynamics of natural populations of Drosophila and their parasitoids.

\section{Abiotic Factors}

The clines and patterns illustrated in figures 1-3 occur over a very broad geographic range encompassing great variation in abiotic factors. Might other selection pressures, unrelated to parasitism itself, influence levels of virulence and resistance? For example, it has often been observed that encapsulation is more effective at higher temperatures (Salt 1970), a generalization that normally, though not always, applies to Drosophila (Fellowes et al., in press). It is possible that this may influence optimum investment in resistance in areas of different average temperature.

As described earlier, the process of encapsulation involves the deposition of melanin, which is also a major part of puparium formation. Mollema (1988) has suggested that in drier climates Drosophila may be selected to have thicker puparia to prevent desiccation and as a result may invest more in the biochemical pathways associated with melanin production. If these pathways are also important in encapsulation, then the relative costs of resistance may be reduced. There is some evidence for a positive geographic correlation between puparial thickness and encapsulation (Kraaijeveld 1994), though the importance of desiccation as a selection pressure in the wild is not known. At least in the laboratory, flies have a high survival rate at humidities as low as $12 \%$, lower than likely to occur in the field, and survival appears not to be influenced by whether the pupae contains an encapsulated parasitoid (M. D. E. Fellowes and P. Masnatta, unpublished data).

\section{The Host-Natural Enemy Community}

A host is seldom attacked by the same parasitoid species complex over its complete geographic range, and, similarly, many if not most parasitoids have host ranges that vary over space. To what extent may changes in the host-parasitoid community be responsible for the geographic patterns in figures 1-3? We first explore the extent of crossresistance (no data yet exist on cross-virulence), then ask whether the distribution of alternative hosts and parasitoids help explain the geographic patterns in resistance and virulence. We finish by examining the role of pupal parasitoids and other natural enemies.

Cross-Resistance. Just as genotype-specific resistance and virulence mechanisms lead to a constant turnover of gene frequencies, so species-specific resistance and virulence may lead to changes in species frequency. We have described earlier artificial selection experiments that created replicate lines of $D$. melanogaster with higher resistance against A. tabida and L. boulardi. Fellowes et al. (in press) measured the resistance of each set of selected lines against the alternative parasitoid. The $L$. boulardi-selected lines performed significantly better than controls against $A$. $t a-$ bida, doing as well as the actual A. tabida-selected lines (fig. 7). However, the performance of the $A$. tabida-selected lines against $L$. boulardi was very poor and not significantly different from the controls. We have recently obtained cultures of an Australian species, Asobara persimilis, that attacks a wide range of host species (Prince 1976). This species has high virulence, and we find that lines selected for resistance against $A$. tabida have low rates of encapsulation against this species, with no difference between selected and control lines (logistic regression, correcting for overdispersion, $\chi_{1}^{2}=1.32$, NS).

In summary, in no cases did we find a decrease in resistance against one parasitoid to be a correlated response to increased resistance against another. Instead, improved resistance against one parasitoid often increased performance against another. However, this may not be symmetrical. For example selection for $L$. boulardi resistance confers A. tabida resistance but not vice versa, which suggests a mixture of parasitoid specific and nonspecific components to resistance. It may be significant that a specific component seems to be required to overcome the specialist L. boulardi.

Cross-Species and Geographic Patterns. In cross-species comparisons, Drosophila differ markedly in their ability to encapsulate parasitoids. Part of this variation may be due to the density of circulating hemocytes, which Eslin and Prévost $(1996,1998)$ have found to be correlated with encapsulation ability. A number of species appear never to encapsulate parasitoids. These include the common north European species Drosophila subobscura, which has never been recorded to encapsulate any of the native parasitoids that attack it or a range of exotic parasitoids in the laboratory (Kraaijeveld and van der Wel 1994). This observation may be very important in determining the cline in A. tabida virulence shown in figure 1 (Kraaijeveld and van Alphen 1994). In northern Europe, D. subobscura is the main host of A. tabida, whereas in the south, where 


\section{Shaded bars - selected lines}

Unshaded bars - control lines
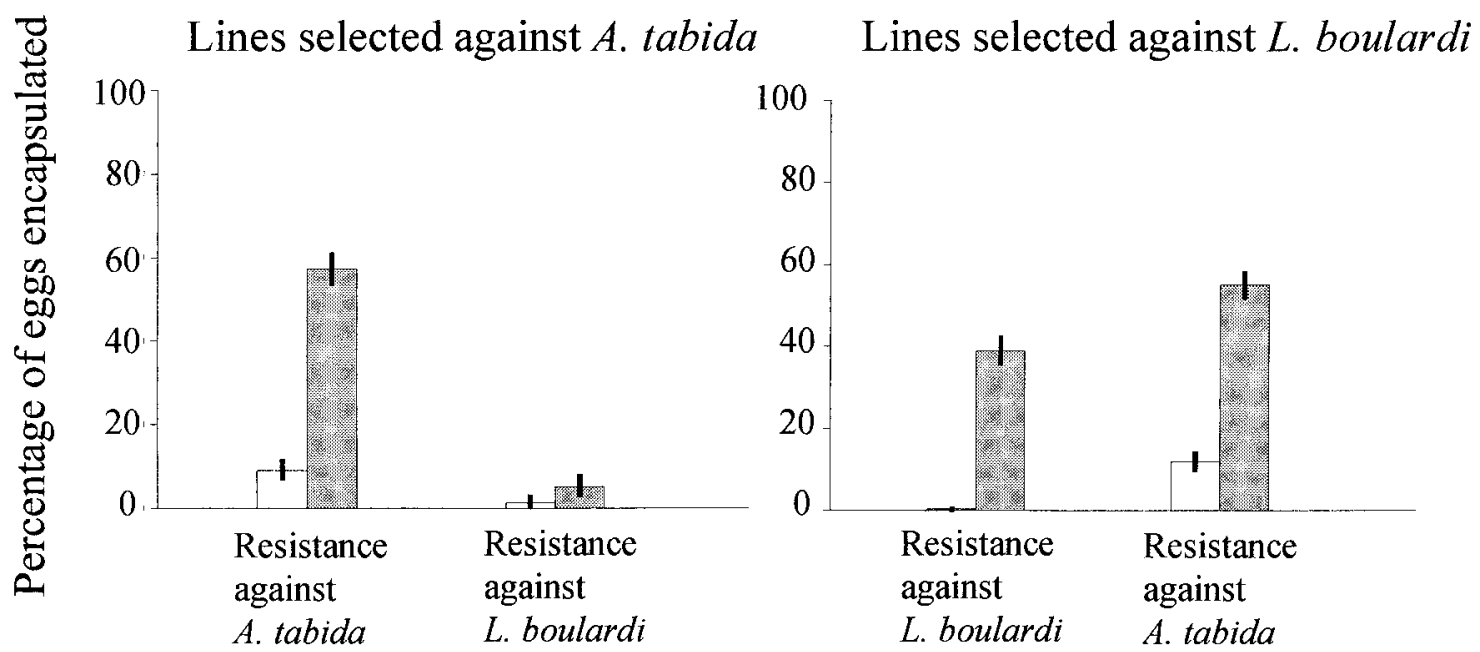

Figure 7: The encapsulation ability of Drosophila melanogaster lines selected for improved resistance against either Asobara tabida (left) or Leptopilina boulardi (right) when tested against both species. Means and SEs of replicate lines are given. There is a strongly significant difference between control and selection lines in all cases except A. tabida lines challenged by L. boulardi, which are not significant.

D. subobscura is much rarer, the main host is D. melanogaster. The pattern is consistent, with A. tabida evolving weak virulence when the most important host is the nondefended D. subobscura and strong virulence when the most important host is the better-defended D. melanogaster.

There is a correlation between host acceptance behavior and virulence in A. tabida (Mollema 1991; Kraaijeveld et al. 1995). Females of low-virulence strains tend to reject D. melanogaster larvae, on which they have low survival, when they are offered in combination with $D$. subobscura larvae, whereas high-virulence strains show little preference. This difference in host selection pressures is at least partially genetically determined.

The relative availability of $D$. subobscura can also explain the exceptions to the general geographic trend. Habitats associated with humans such as shops, markets, and urban areas support higher populations of D. melanogaster and hence may select for greater A. tabida virulence. However, the virulent strain from the Scottish sherry casks is still unexplained because the only host species present was $D$. ambigua (A. Davis, personal communication), which appears, like D. subobscura, not to encapsulate parasitoids. The host of the Canadian A. tabida strain of intermediate virulence is Drosophila athabasca, for which Streams (1968) reported a weak encapsulation response, again con- sistent with the hypothesis (though note that his flies came from a different locality).

The importance of host range is supported by observations of Asobara rufescens, a sibling species of A. tabida. In northern Europe, $A$. rufescens occurs exclusively on decaying plant material where it mainly attacks the larvae of Drosophila fenestrarum and the drosophilid Scaptomyza pallida (Vet and Janse 1984). These species have never been recorded as encapsulating parasitoid eggs, and A. rufescens from northern Europe has low virulence against D. melanogater when persuaded to attack this species in the laboratory. In southern Europe, however, A. rufescens also attacks fruit where it encounters the more resistant $D$. melanogaster, and stains of the parasitoid from these areas have significantly higher virulence against $D$. melanogaster (Kraaijeveld et al. 1994).

Could the presence of A. tabida as the major parasitoid of $D$. melanogaster also explain patterns in the resistance of the latter? Up to a point: D. melanogaster resistance is low in northern Europe where A. tabida attacks $D$. subobscura and low in the Iberian peninsula where A. tabida is absent. But it is also low in southeastern Europe where A. tabida is present and believed to attack D. melanogaster. Clearly other factors must also be involved.

Pupal Parasitoids. Could patterns of host resistance be in- 
fluenced by the other guild of parasitoids that attack the pupae? The parasitoids that attack Drosophila pupae typically attack a wide range of dipteran species (Nøstvik 1954) and are in fact mostly ectoparasitoids: they lay their eggs inside the host puparium but outside the actual pupa. Therefore, the eggs do not come into contact with the host's immune system. Because pupal parasitoids have to drill through the puparial wall to lay their eggs, the latter constitutes the host's main defense. Both puparium formation and encapsulation share at least partly the same metabolic requirements, at both a cellular and a biochemical level (Fraenkel and Rudall 1947; Nappi and Streams 1969; Rizki et al. 1980; see also our earlier discussion). There is thus the possibility that resistance mechanisms against larval parasitoids may improve defense against pupal parasitoids and vice versa.

Kraaijeveld (1994) found geographic variation in puparium thickness and a weak, but significant, positive correlation between puparium thickness and encapsulation ability against $A$. tabida across 17 European populations. However, there was no significant difference in the thickness of the puparial wall in D. melanogaster lines selected for resistance against A. tabida and their controls (A. R. Kraaijeveld, unpublished data), while Delpuech et al. (1994) found no correlation between resistance to the pupal parasitoid Pachycrepoidus vindemmiae and Leptopilina species.

Other Natural Enemies. Moving beyond parasitoids, might we find that other natural enemies influence patterns of resistance and virulence? Drosophila are parasitized by a number of allantonematid nematode species that live internally in the hemocoel. The best known is Howardula aoronymphium, which occurs in Europe and North America and attacks larvae of the quinaria group and other mycophagous species (Welch 1959; Jaenike 1992). In North America, Drosophila neotestacea is reported encapsulating H. aoronymphium (Jaenike 1992), and hence nematodes are a potential selection pressure on the encapsulation system. Parasitylenchus diplogenus occurs on fermenting substrates in Europe and parasitizes species of the obscura group and possibly D. melanogaster (Welch 1959).

Little is known about the abundance of $P$. diplogenus. In a study in the Netherlands, rates of parasitism of $D$. subobscura varied between $0 \%$ and 10\% (Gillis and Hardy 1997). Similar low values were found during a 2-yr survey of obscura group flies in southern England (A. R. Kraaijeveld, unpublished data). The wider geographic distribution of $P$. diplogenus is as yet unknown. Whether increased resistance against parasitoids influences defense against microparasites such as fungi, bacteria, and viruses attacking Drosophila larvae remains to be seen.

\section{Conclusions}

Parasitoids develop at the expense of their host and hence have been subject to intense selection pressure to overcome host defenses. Many of the sophisticated adaptations that have evolved are now understood at a physiological and increasingly at a molecular level (Salt 1970; Beckage et al. 1993; Strand and Pech 1995). The basic humoral and cellular defense mechanisms of the host evolved before the appearance of parasitoids and are a general defense mechanism against a wide range of challenges (Gillespie et al. 1997). Nevertheless, the imprint of parasitoid selection can be seen in the fine-tuning of the host defenses (Salt 1970; Beckage et al. 1993; Strand and Pech 1995), and artificial selection experiments show that parasitoids can select for changes in the level of host defenses. There is thus clear evidence that host resistance has influenced parasitoid virulence and vice versa.

Is there evidence of specific, coupled coevolution between pairs of species? Depending on the nature of resistance and virulence, such coupling could give rise to cycles in gene frequency and Red Queen coevolution, or it might lead to locally fixed optima for the levels of resistance and virulence, which may vary across time or space as costs and benefits change. Though more work is required, the available information to date suggests that resistance and virulence are quantitative rather than qualitative traits, by which we mean that increased resistance improves survival against all genotypes of a parasitoid, and increased virulence improves performance against all genotypes of host. Though the relevant experiments have yet to be done, we conjecture that the rank order of survival of different host strains exposed to different parasitoid strains will be constant. This lack of frequency dependence suggests to us that based on present evidence Red Queen coevolution involving changes in virulence and resistance (Lively 1999, in this issue) is unlikely in host-parasitoid systems.

Coupled coevolution could still lead to spatiotemporal variation in resistance and virulence. Because parasitoids often have a major effect on host population dynamics, there is the possibility that higher parasitoid densities may lead to selection for enhanced resistance, which might then cause either a reduction in parasitoid numbers (and hence a relaxation of selection for resistance) or a corresponding increase in the level of virulence. Preliminary modeling of this type of interaction that assumes density-dependent rather than frequency-dependent selection suggests that the population and genetic dynamics may reach an equilibrium or show persistent cycles, depending on the initial conditions (Sasaki and Godfray 1999). Unfortunately, unlike in the rust-plant system of Burdon and Thrall (1999, in this issue), there are as yet no long-term studies of resistance and virulence levels with which to test these 
ideas. One fact that argues against the importance of this type of temporal cycle is the sparse evidence for additive genetic variation in Drosophila parasitoids (but see Henter 1995). However, the recent demonstration of costs of increased resistance that are only manifest at high levels of intraspecific competition suggests another possible source of density-dependent selection still to be explored theoretically.

The geographic patterns described at the beginning of this article may be influenced by coupled interactions between hosts or parasitoids, but at present the most likely explanation seems to be geographic difference in hostparasitoid community structure. The clearest pattern is the north-south cline in Asobara tabida virulence: this is most easily explained by lower virulence occurring when the main host is the poorly defended Drosophila subobscura and higher virulence evolving when Drosophila melanogaster is the most important host. There is a trend for $D$. melanogaster resistance to this species to be higher where virulent $A$. tabida strains occur (as happens in the laboratory), but this observation requires confirmation and does not explain the complete pattern. Thus, the bestsupported hypothesis for the geographic pattern of virulence requires just parasitoid adaptation rather than coevolution. Whether abiotic factors also play a significant role in this remains to be seen.

Although we argue that Drosophila and its parasitoids are an attractive system for studying coevolutionary interactions, this work is still very much in its infancy. We finish here by highlighting areas that need more study. First, confirmation is needed of the apparent lack of genetic variation for parasitoid virulence compared with host resistance and of the conjecture that resistance and virulence are ordinal traits. The possibility of coupled coevolution could be explored by comparing the evolution of resistance and virulence in population cages in which both hosts and parasitoids are free to evolve with the response of each party in artificial selection experiments. An experimental and comparative approach could also be used to investigate the effect of community structure on the two traits. An interesting question here is to explain why some hosts, such as D. subobscura, seem never to defend themselves against parasitoids. Working with Drosophila offers great possibilities for investigating the genetics of adaptation.

A problem with resistance and virulence is that they are traits that cannot be measured on individuals, at least at present. This point makes genetic investigation difficult, though we are currently exploring a number of physiological correlates of resistance that can be measured in single flies. Already a number of genes have been identified in isofemale line studies that are related to resistance and virulence (Carton et al. 1992; Carton and Nappi 1997; Orr and Irving 1997; Benassi et al. 1998; Dupas et al. 1998), though this technique does not necessarily reveal the genes responsible for variation in the field. However, Orr and Irving (1997) used classical genetic techniques to localize the difference in resistance to $A$. tabida between southern and northern $D$. melanogaster strains to the centromere region of chromosome 2, strongly suggestive of a simple genetic basis. They also point out the value of Drosophila resistance as a model system with which to investigate the genetics of adaptation.

Finally, one of the greatest challenges will be to integrate the genetics, evolutionary biology, and population dynamics into models of resistance and virulence that can generate hypotheses that can be tested in the field and laboratory.

\section{Acknowledgments}

This article is part of a working group supported by the National Center for Ecological Analysis and Synthesis (NCEAS; National Science Foundation grant DEB9421535) and was cofunded by the American Society of Naturalists and NCEAS. Some of the work was supported by core funding to the Natural Environment Research Council Centre for Population Biology. J. van Alphen, G. Boskamp, and J. Ellers kindly provided the Asobara persimilis strain and the Saanich strain of Asobara tabida. We are grateful to D. Althoff, M. Fellowes, and J. van Alphen for valuable discussions and comments on previous versions of the article.

\section{Literature Cited}

Atkinson, W. D. 1979. A field investigation of larval competition in domestic Drosophila. Journal of Animal Ecology 48:91-102.

Bailey, V. A., A. J. Nicholson, and J. Williams. 1962. Interaction between hosts and parasites when some host individuals are more difficult to find than others. Journal of Theoretical Biology 3:1-18.

Beckage, N. E., S. N. Thompson, and B. A. Federici. 1993. Parasites and pathogens of insects. Vol 1. Parasites. Academic Press, San Diego, Calif.

Benassi, V., F. Frey, and Y. Carton. 1998. A new specific gene for wasp cellular immune resistance in Drosophila. Heredity 80:347-352.

Boulétreau, M. 1986. The genetic and coevolutionary interaction between parasitoids and their hosts. Pages 169-195 in J. K. Waage and D. J. Greathead, eds. Insect parasitoids: thirteenth symposium of the Royal Entomological Society of London. Academic Press, London.

Boulétreau, M., and P. Fouillet. 1982. Variabilité génétique intrapopulation de l'adéquation de Drosophila melan- 
ogaster à un de ses parasites hyménoptères. Compte Rendu de l'Académie des Sciences 295:775-778.

Burdon, J. J., and P. H. Thrall. 1999. Spatial and temporal patterns in coevolving plant and pathogen associations. American Naturalist 153(suppl.):S15-S33.

Carton, Y. 1984. Analyse expérimentale de trois niveaux d'interactions entre Drosophila melanogaster et le parasite Leptopilina boulardi (sympatrie, allopatrie, xénopatrie). Génétique, Sélection et Evolution 16:417-430.

Carton, Y., and M. Boulétreau. 1985. Encapsulation ability of Drosophila melanogaster: a genetic analysis. Developmental and Comparative Immunology 9:211-219.

Carton, Y., and J. R. David. 1983. Reduction of fitness in Drosophila adults surviving parasitization by a cynipid wasp. Experientia 39:231-233.

Carton, Y., and A. J. Nappi. 1991. The Drosophila immune reaction and the parasitoid capacity to evade it: genetic and coevolutionary aspects. Acta Oecologica 12:89-104.

- 1992. Methods for genetic investigation of cellular immune reaction in insects, with the parasitic wasp (Drosophila) system as a model. Pages 92-101 in J. P. N. Pathak, ed. Insect immunity. IBH, Oxford.

- 1997. Drosophila cellular immunity against parasitoids. Parasitology Today 13:218-227.

Carton, Y., M. Boulétreau, J. J. M. van Alphen, and J. C. van Lenteren. 1986. The Drosophila parasitic wasps. Pages 347-394 in M. Ashburner, H. L. Carson, and J. N. Thompson, eds. The genetics and biology of Drosophila. Vol. 3. Academic Press, London.

Carton, Y., P. Capy, and A. J. Nappi. 1989. Genetic variability of host-parasite relationship traits: utilization of isofemale lines in a Drosophila simulans parasitic wasp. Génétique, Sélection et Evolution 21:437-446.

Carton, Y., F. Frey, and A. J. Nappi. 1992. Genetic determinism of the cellular immune reaction in Drosophila melanogaster. Heredity 69:393-399.

Delpuech, J.-M., F. Frey, and Y. Carton. 1994. Genetic and epigenetic variation in suitability of a Drosophila host to three parasitoid species. Canadian Journal of Zoology 72:1940-1944.

Doebeli, M. 1997. Genetic variation and the persistence of predator-prey interactions in the Nicholson-Bailey model. Journal of Theoretical Biology 188:109-120.

Driessen, G., L. Hemerik, and J. J. M. van Alphen. 1990. Drosophila species, breeding in the stinkhorn (Phallus impudicus Pers.) and their larval parasitoids. Netherlands Journal of Zoology 40:409-427.

Dupas, S., M. Brehelin, F. Frey, and Y. Carton. 1996. Immune suppressive virus-like particles in a Drosophila parasitoid: significance of their intraspecific morphological variations. Parasitology 113:207-212.

Dupas, S., F. Frey, and Y. Carton. 1998. A single parasitoid segregating factor controls immune suppression in Drosophila. Journal of Heredity 89:306-311.

Eslin, P., P. Giordanengo, Y. Fourdrain, and G. Prévost. 1996. Avoidance of encapsulation in the absence of VLP by a braconid parasitoid of Drosophila larvae: an ultrastructural study. Canadian Journal of Zoology 74: 2193-2198.

Eslin, P., and G. Prévost. 1996. Variation in Drosophila concentration of haemocytes associated with different ability to encapsulate Asobara tabida larval parasitoid. Journal of Insect Physiology 42:549-555.

. 1998. Hemocyte load and immune resistance to Asobara tabida are correlated in species of the Drosophila melanogaster subgroup. Journal of Insect Physiology 44: 807-816.

Fellowes, M. D. E., P. Masnatta, A. R. Kraaijeveld, and H. C. J. Godfray. 1998a. Pupal parasitoid attack influences the relative fitness of Drosophila that have encapsulated larval parasitoids. Ecological Entomology 23:281-284.

Fellowes, M. D. E., A. R. Kraaijeveld, and H. C. J. Godfray. 1998b. Trade-off associated with selection for increased ability to resist parasitoid attack in Drosophila melanogaster. Proceedings of the Royal Society of London B, Biological Sciences 265:1553-1558.

- 1999. The relative fitness of Drosophila melanogaster (Diptera, Drosophilidae) that have successfully defended themselves against the parasitoid Asobara tabida (Hymenoptera, Braconidae). Journal of Evolutionary Biology 12:123-128.

- In press. Cross-resistance following artificial selection for increased defence against parasitoids in Drosophila melanogaster. Evolution.

Fraenkel, G., and K. M. Rudall. 1947. The structure of insect cuticles. Proceedings of the Royal Society of London B, Biological Sciences 134:111-143.

Gillespie, J., M. R. Kanost, and T. Trenczek. 1997. Biological mediators of insect immunity. Annual Review of Entomology 42:611-643.

Gillis, J. E. M., and I. C. W. Hardy. 1997. Nematode parasitism in a northern European drosophilid community. Entomologia Experimentalis et Applicata 84:275-291.

Godfray, H. C. J. 1994. Parasitoids: behavioural and evolutionary ecology. Princeton University Press, Princeton, N.J.

Godfray, H. C. J., and M. P. Hassell. 1991. Encapsulation and host-parasitoid population biology. Pages 131-147 in C. A. Toft, A. Aeschlimann, and L. Bolis, eds. Parasitehost associations: coexistence or conflict? Oxford University Press, Oxford.

Godfray, H. C. J., and C. B. Müller. 1998. Host-parasitoid dynamics. Pages 135-166 in J. P. Dempster and I. Maclean, eds. Insect populations: in theory and practice. Kluwer, Dordrecht. 
Hadorn, E., and A. Graßmann. 1962. Drosophila und Pseudeucoila. IV. Artspezifische Unterschiede in der Abwehrreaktion auf verschieden resistente Wespenstämme. Schweizerische Gesellschaft für Vererbungsforschung Jahresbericht 37:21-27.

Hadorn, E., and I. Walker. 1960. Drosophila und Pseudeucoila. I. Selektionsversuch zur Steigerung der Abwehrreaktion des Wirtes gegen den Parasiten. Revue Suisse de Zoologie 67:216-225.

Hassell, M. P. 1978. The dynamics of arthropod predatorprey systems. Princeton University Press, Princeton, N.J.

- 1998. The dynamics of host-parasitoid interactions. Oxford University Press, Oxford.

Hassell, M. P., and R. M. Anderson. 1984. Host susceptibility as a component in host-parasitoid systems. Journal of Animal Ecology 53:611-621.

Henter, H. J. 1995. The potential for coevolution in a hostparasitoid system. II. Genetic variation within a population of wasps in the ability to parasitize an aphid host. Evolution 49:439-445.

Henter, H. J., and S. Via. 1995. The potential for coevolution in a host-parasitoid system. I. Genetic variation within an aphid population in susceptibility to a parasitic wasp. Evolution 49:427-438.

Hochberg, M. E., and R. D. Holt. 1995. Refuge evolution and the population dynamics of coupled host-parasitoid associations. Evolutionary Ecology 9:633-661.

Hughes, K., and M. B. Sokolowski. 1996. Natural selection in the laboratory for a change in resistance by Drosophila melanogaster to the parasitoid wasp Asobara tabida. Journal of Insect Behavior 9:477-491.

Jaenike, J. 1992. Mycophagous Drosophila and their nematode parasites. American Naturalist 139:893-906.

Janssen, A. R. M., G. Driessen, M. de Haan, and N. Roodbol. 1988. The impact of parasitoids on natural populations of temperate woodland Drosophila. Netherlands Journal of Zoology 38:61-73.

Kraaijeveld, A. R. 1994. Local adaptations in a parasitoidhost system: a coevolutionary arms race? Ph.D. diss. University of Leiden, Leiden.

Kraaijeveld, A. R., and H. C. J. Godfray. 1997. Trade-off between parasitoid resistance and larval competitive ability in Drosophila melanogaster. Nature (Paris) 389: 278-280.

Kraaijeveld, A. R., and J. J. M. van Alphen. 1994. Geographic variation in resistance of the parasitoid Asobara tabida against encapsulation by Drosophila melanogaster: the mechanism explored. Physiological Entomology 19: 9-14.

- 1995. Geographic variation in encapsulation ability of Drosophila melanogaster and evidence for parasitoid-specific components. Evolutionary Ecology 9: 10-17.
Kraaijeveld, A. R., and N. N. van der Wel. 1994. Geographic variation in reproductive success of the parasitoid Asobara tabida in larvae of several Drosophila species. Ecological Entomology 19:221-229.

Kraaijeveld, A. R., S. Voet, and J. J. M. van Alphen. 1994. Geographic variation in habitat choice and host suitability in the parasitoid Asobara rufescens. Entomologia Experimentalis et Applicata 72:109-114.

Kraaijeveld, A. R., B. Nowee, and R. W. Najem. 1995. Adaptive variation in host selection behaviour of Asobara tabida, a parasitoid of Drosophila larvae. Functional Ecology 9:113-118.

Kraaijeveld, A. R., J. J. M. van Alphen, and H. C. J. Godfray. 1998. The coevolution of host resistance and parasitoid virulence. Parasitology 116:S29-S45.

Lande, R. 1976. Natural selection and random genetic drift in phenotypic evolution. Evolution 30:314-334.

Lively, C. 1999. Migration, virulence, and the geographic mosaic of adaptation by parasites. American Naturalist 153(suppl.):S34-S47.

Mills, N. J., and W. M. Getz. 1996. Modelling the biological control of insect pests: a review of host-parasitoid models. Ecological Modelling 92:121-143.

Mollema, C. 1988. Genetical aspects of resistance in a hostparasitoid interaction. Ph.D. diss. University of Leiden, Leiden.

- 1991. Heritability estimates of host selection behaviour by the Drosophila parasitoid Asobara tabida. Netherlands Journal of Zoology 41:174-183.

Murdoch, W. W., and C. J. Briggs. 1996. Theory for biological control: recent developments. Ecology 77: 2001-2013.

Nappi, A. J., and F. A. Streams. 1969. Haemocytic reactions of Drosophila melanogaster to the parasites Pseudeucoila mellipes and P. bochei. Journal of Insect Physiology 15: 1551-1566.

Nøstvik, E. 1954. Biological studies of Pachycrepoideus dubius Ashmead (Chalcidoidea: Pteromalidae), a pupal parasite of various Diptera. Oikos 5:195-204.

Oprecht, E., and E. Hadorn. 1952. Stock-specific defense reactions against a parasite. Drosophila Information Service 26:116.

Orr, H. A., and S. Irving. 1997. The genetics of adaptation: the genetic basis of resistance to wasp parasitism in Drosophila melanogaster. Evolution 51:1877-1885.

Parsons, P. A. 1980. Isofemale strains and evolutionary strategies in natural populations. Pages 175-217 in $\mathrm{H}$. Hecht, W. Steere, and B. Wallace, eds. Evolutionary biology. Plenum, New York.

Powell, J. R. 1997. Progress and prospects in evolutionary biology: the Drosophila model. Oxford University Press, Oxford.

Prince, G. J. 1976. Laboratory biology of Phaenocarpa per- 
similis Papp (Braconidae; Alysiinae), a parasitoid of Drosophila. Australian Journal of Zoology 24:249-264.

Rizki, R. M., and T. M. Rizki. 1990. Parasitoid virus-like particles destroy Drosophila cellular immunity. Proceedings of the National Academy of Sciences of the USA 87:8388-8392.

Rizki, T. M., R. M. Rizki, and E. H. Grell. 1980. A mutant affecting the crystal cells in Drosophila melanogaster. William Roux's Archive of Developmental Biology 188: 91-99.

Salt, G. 1970. The cellular defence reactions of insects. Cambridge University Press, Cambridge.

Sasaki, A., and H. C. J. Godfray. 1999. A model for the coevolution of resistance and virulence in coupled hostparasitoid interactions. Proceedings of the Royal Society of London B, Biological Sciences 266:455-463.

Schlegel-Oprecht, E. 1953. Versuche zur Auslösung von Mutationen bei der zoophagen Cynipide Pseudeucoila bochei Weld und Befunde über die stammspezifische Abwehrreaktion des Wirtes Drosophila melanogaster. Zeitschrift für Induktive Abstammungs- und Vererbungslehre 85:245-281.

Strand, M. R., and L. L. Pech. 1995. Immunological basis for compatibility in parasitoid-host relationships. Annual Review of Entomology 40:31-56.

Streams, F. A. 1968. Defense reactions of Drosophila species (Diptera: Drosophilidae) to the parasite Pseudeucoila bochei (Hymenoptera: Cynipidae). Annals of the Entomological Society of America 61:158-164.

Thompson, J. N. 1982. Interaction and coevolution. Wiley, New York.

1994. The coevolutionary process. University of Chicago Press, Chicago.

. 1999. Specific hypotheses on the geographic mosaic of coevolution. American Naturalist 153(suppl.): S1-S14.

Vet, L. E. M., and C. J. Janse. 1984. Fitness of two sibling species of Asobara (Braconidae; Alysiinae): larval parasitoids of Drosophilidae in different microhabitats. Ecological Entomology 9:345-354.

Wajnberg, E., G. Prévost, and M. Boulétreau. 1985. Genetic and epigenetic variation in Drosophila larvae suitability to a hymenopterous endoparasitoid. Entomophaga 30: 187-191.

Walker, I. 1959. Die Abwehrreaktion des Wirtes Drosophila melanogaster gegen die zoophage Cynipide Pseudeucoila bochei Weld. Revue Suisse de Zoology 66:569-632.

1962. Drosophila und Pseudeucoila. III. Selektionsversuche zur Steigerung der Resistenz des Parasiten gegen die Abwehrreaktion des Wirtes. Revue Suisse de Zoology 69:209-227.

Welch, H. E. 1959. Taxonomy, life cycle, development, and habits of two new species of Allantonematidae $(\mathrm{Ne}-$ matoda) parasitic in drosophilid flies. Parasitology 49: 3-103. 